

\title{
ESPACIALIZAÇÃO DAS OCORRENCIAS DA COMPANHIA DE POLÍCIA DE PROTEÇÃO AMBIENTAL - COPPA, ATRAVÉS DO USO DE GEOTECNOLOGIAS
}

\author{
Rosana Maria Santos Sant"Ana \\ Silvana Sá de Carvalho \\ Arnaldo Bispo de Jesus
}

p. $71-81$

Como citar este artigo: SANT"ANA, R. M. S. ESPACIALIZAÇÃO DAS OCORRENCIAS DA COMPANHIA DE POLÍCIA DE PROTEÇÃO AMBIENTAL - COPPA, ATRAVÉS DO USO DE GEOTECNOLOGIAS. Revista Eletrônica: Tempo - Técnica - Território, v.5, n.1 (2014), p. $71: 81$ ISSN: $2177-4366$.

DOI: https://doi.org/10.26512/ciga.v5i1.22151

Disponível em: http://periodicos.unb.br/index.php/ciga/

$$
\mathrm{T}-\mathrm{T}-\mathrm{T}
$$

Revista Eletrônica:

Tempo - Técnica - Território, V.5, N.1 (2014), 71:81 ISSN : $2177-4366$

DOI: https:// doi.org/10.26512/ ciga.v5i1.22151 


\title{
ESPACIALIZAÇÃO DAS OCORRENCIAS DA COMPANHIA DE POLÍCIA DE PROTEÇÃO AMBIENTAL - COPPA, ATRAVÉS DO USO DE GEOTECNOLOGIAS
}

\author{
Rosana Maria Santos Sant”Ana \\ Curso de Especialização em Geotecnologias - Escola de Engenharia Eletro-Mecânica da Bahia, \\ EEMBA. E-mail: rmssantana@hotmail.com
}

\begin{abstract}
Silvana Sá de Carvalho
Programa de Pós-Graduação em Planejamento Territorial e Desenvolvimento Social - Universidade Católica do Salvador, UCSal. E-mail: silvanasc@ucsal.br
\end{abstract}

\author{
Arnaldo Bispo de Jesus \\ Curso Sistemas da Informação - Universidade Católica do Salvador, UCSal. E-mail: \\ santobispo@gmail.com
}

\begin{abstract}
Resumo : O objetivo deste trabalho é mostrar a distribuição espacial das ocorrências registadas na Companhia de Policia e Proteção Ambiental - COPPA, em Salvador e mais 12 municípios da Região Metropolitana, no ano de 2012, utilizando as Geotecnologias. Inicialmente foi feita a coleta dos dados, através da digitalização das fichas de ocorrência da COPPA em um Sistema de Informações Geográficas - WEB (SIG-WEB), e como esses dados continham a localização geográfica da ocorrência, foi possível especializa-los e sistematiza-los num software específico de Geoprocessamento - o ArcGIS. Posteriormente, foi aplicada a Estimativa de Densidade de Kernel para poder visualizar onde há maior concentração desses pontos, então foi possível identificar e analisar áreas onde ocorre o maior número de solicitações para atender chamados de insetos sociais, animais silvestres e também denúncias de crimes ambientais. Espera-se que os mapas resultantes possam oferecer um apoio para o planejamento de ações desta Companhia.
\end{abstract}

Palavras-chave: Polícia Ambiental, Geotecnologias, Mapa de Kernel.

\begin{abstract}
The purpose of this article is to show the spatial distribution of occurrences registered in Environmental Police Company in Salvador and more 12 cities of its Metropolitan Region in the year 2012, using Geotechnologies. Initially, data was collected and inserted into a Geographical Information System-WEB (GIS-WEB). These records contained a nearly geographic location of the occurrence. Thus it was possible to specalize them and systemaize them using an specific geoprocesing software - the ArcGIS. Lately the Kernel density Estimator was apllied to data to become possible visualizing the spots of higher concentration of point. Then it was possible to idenify and analyze areas with larger rates of requests upon social insects, sylvan animals and even environmental crimes denounces. It is expected that the resulting maps can give a support on the planning of actions to this company.
\end{abstract}

Key-words: Environmental police, Geotechnology, Kernel Map. 
Résumé: L'objectif de ce travail est de montrer la répartition spatiale d'occurrences enregistrées dans la compagnie de police de protection de l'environnement - COPPA, à Salvador et en 12 municipalités de la région métropolitaine, en l'an de 2012, à l'aide de la géotechnique. Initialement a été fait un recueille des informations par la digitalization des dossiers d'occurrences de la COPPA dans un Système d'Information Géographique - WEB (Web-SIG), et comment ces informations contenaient la localisation géographique de l'occurrence, il était possible de les spécialiser et de les systématiser dans un logiciel spécifique de géotraitement SIG - ArcGIS. Par la suite, cela a appliquée un montant estime de densité de Kernel pour pouvoir visualiser où il y a une plus grande concentration de ces points, ainsi c'était possible d'identifier et d'analyser les zones où les demandes pour répondre aux appels d'occurrences d'insectes sociaux, des animaux sauvages et aussi des allégations de crimes contre l'environnement étaient plus grandes. Il est prévu que les cartes qui en résultent puissent fournir un soutien pour la planification des actions de la Compagnie.

Mots-clés: police de l'environnement, la géotechnique, la carte de Kernel.

\section{INTRODUÇÃO}

O uso de Geotecnologias está cada vez mais frequente dentro de várias áreas como saúde, meio ambiente, geomarketing, gestão municipal, transporte, agricultura, segurança, e muitas outras que se beneficiam com as ferramentas oferecidas pelo Geoprocessamento.

Essas ferramentas computacionais para Geoprocessamento, também chamadas de Sistemas de Informações Geográficas (SIGs), consistem em um conjunto de elementos orientados para o processamento e análise de dados com referência espacial. Portanto, o SIG é um suporte operacional que materializa o Geoprocessamento. Torna-se uma ferramenta importante, pois a partir de espaços mapeados, pode-se conhecer melhor uma área, possibilitando assim, o fornecimento de subsídios para uma futura tomada de decisões.

Tanto na administração pública ou privada, o SIG já é uma realidade. Esses ambientes podem ser utilizados como polos geradores de informações, imprescindíveis na utilização do planejamento e funcionamento de instituições/órgãos específicos, como por exemplo, a segurança pública.

A introdução do SIG na segurança pública veio para substituir os mapas de pinos, que com uma simples inspeção visual é possível enxergar a correlação entre os fenômenos numa mesma área (SAURET, 2012, p.72).

Diante da importância do uso das Geotecnologias e atrelado a isso, a necessidade de planejar e executar ações que melhorem o desempenho de algumas Companhias especializadas, o principal objetivo deste projeto é a utilização do SIG para espacializar as ocorrências registradas na Companhia de Policia de Proteção Ambiental - COPPA, na cidade se Salvador e sua Região Metropolitana, através do uso de Geotecnologias. Com isso, será possível criar mecanismos que possibilitem novas estratégias de gestão. 
A COPPA recebe diariamente inúmeras ligações para atender ocorrências de animais silvestres e insetos sociais que ‘invadem’ residências. No ano de 2012 mais de 50\% das solicitações foram para atender chamados de ataques de abelhas e marimbondos. A maioria desses deslocamentos de insetos ocorre em períodos quentes, porque saem em busca de lugares mais frios. Ao contrário dos animais silvestres, como as serpentes, que saem em busca de calor.

A COPPA é subordinada ao Comando de Policiamento Especializado e foi criado em 8 de agosto de 1979. No estado da Bahia, ela é a responsável pelo policiamento ostensivo ambiental fardado, preventivo e repressivo, visando à preservação da vida no meio ambiente em todo território.

Apesar de haver equipes bem definidas para cada atividade, a COPPA tem inúmeras dificuldades para realização de suas atividades diárias. São problemas operacionais e gerenciais que dificultam a adoção de medidas eficientes e eficazes para agilizar os trabalhos direcionados a esta Companhia.

\section{MATERIAIS E MÉTODOS}

A elaboração deste projeto ocorreu em diversas etapas. Inicialmente foi feito a coleta de dados, através de fichas fornecidas pela COPPA. Essas fichas contem todas as ocorrências solicitadas a COPPA durante o ano de 2012, com as seguintes informações: data da solicitação, quem solicita, tipo de ocorrência, endereço e alguma referencia deste endereço. As fichas foram digitadas num SIG-WEB produzido no âmbito do projeto Sistema de Informações Geográficas Aplicado ao Policiamento Ambiental (SIGAPA) executado pela Universidade Católica do Salvador (UCSal) em parceria com a COPPA, financiado pela Fundação de Amparo à Pesquisa do Estado da Bahia (FAPESB).

Com a digitação das fichas, as informações foram anexadas a um banco de dados no qual posteriormente foi gerado um $d b f$. No total foram registradas 1.005 solicitações a COPPA no ano de 2012.

Após a geração deste $d b f$ foi feito o tratamento destes dados. Verificou-se algumas inconsistências no banco de dados, com a ajuda de agentes da COPPA. Há ocorrências solicitadas a COPPA em que foram registradas mais de uma vez para o mesmo local, pelo mesmo solicitante ou por outros. Por isso, a duplicidade de várias ocorrências foi corrigida, restando na base de dados 914 ocorrências.

É importante salientar que as ocorrências foram divididas em três tipos: insetos sociais, animais silvestres e denuncias. Das 914 ocorrências, 481 foram sobre insetos sociais, 250 animais silvestres e 183 denúncias. 
Durante a digitação destas informações, o SIG-WEB permitiu acessar o site Google Maps, onde foi capturada a coordenada geográfica, do centroide de cada logradouro onde foi solicitado o atendimento das ocorrências. De algumas destas ocorrências foi possível identificar pontos mais exatos de sua localização, através de referencias anexadas as informações. Logo a coordenada deixa de ser o centroide do logradouro e passa a ser ponto de referencia localizado no site.

Após a sistematização da tabela dbf, foi gerado um arquivo de pontos no formato shp para utilização no software ArcGis, para melhor visualização dos dados.

Quando há necessidade de representar pontualmente a localização de uma ocorrência ou a intensidade de um fenômeno, podem ser apresentados mapas de pontos. A análise de pontos, neste caso, é o mais indicado por se ter a localização pontual do evento, no caso as ocorrências, através das coordenadas. Neste tipo de representação algumas características são importantes como a distância entre os pontos, a densidade dos pontos e sua distribuição espacial.

Diante disso, através de um mapa de pontos é possível calcular a densidade dessas ocorrências. Nesta análise verificou-se que um mapa de densidade seria de extrema importância para avaliar como se dá a espacialização do fenômeno ocorrido. Portanto, optou-se pela Estimativa de Densidade de Kernel, para poder visualizar onde há maior concentração desses pontos.

A Densidade de Kernel é uma técnica de interpolação exploratória que gera uma superfície de densidade para a identificação visual de “áreas quentes”. Entende-se a ocorrência de uma área quente como uma concentração de eventos que indica de alguma forma a aglomeração em uma distribuição espacial. O estimador Kernel não é um método de detecção de aglomerados por si, mas, um método para explorar e mostrar o padrão de pontos de dados das ocorrências, e é útil a partir do momento em que gera uma superfície contínua a partir de dados pontuais (BARCELLOS; SILVA; ANDRADE, 2007).

O estimador Kernel produz uma superfície contínua, com densidades calculadas em todas as localizações. Essa técnica apresenta, como uma das maiores vantagens, a rápida visualização de áreas que merecem atenção. Portanto o estimador de intensidade é uma boa alternativa para se avaliar o comportamento dos padrões de pontos em uma determinada área de estudo, sendo considerado muito útil para fornecer uma visão geral da distribuição de primeira ordem dos eventos. (CÂMARA; CARVALHO, 2004).

Por fim, após esses estudos foram elaborados mapas de densidade com todas as ocorrências, e os outros com as variáveis isoladas (insetos sociais, animais silvestres e denuncias).

\section{RESULTADOS E DISCUSSÕES}


O desmatamento ostensivo ocorrido em Salvador e Região Metropolitana nos últimos anos está fazendo com que animais migrem de seu habitat natural para outros locais, inclusive residências ou áreas bem próximas a elas. Os animais se sentem acuados e deslocam-se sem saber exatamente onde está indo.

De acordo com o levantamento dos dados do ano de 2012, a COPPA registrou 1.005 ocorrências distribuídas em Salvador e Região Metropolitana. Como já informado anteriormente, apenas 914 registros foram selecionados para o estudo desta pesquisa.

Tanto Salvador quanto os outros municípios que compõem a sua Região Metropolitana, constantemente passam por alterações ambientais provocadas, principalmente, pela especulação imobiliária. Com isso, os animais que ali vivem, são obrigados a se adaptarem a outros meios. Isso talvez possa explicar os altos índices de solicitações da população da RMS para captura e manejo de animais silvestres e insetos sociais, registrados pela COPPA.

Através destes registros, acrescentando suas coordenadas, foi possível especializar a distribuição dessas ocorrências, como pode ser observada através de um mapa da RMS na figura 1.

Figura 1 - Mapa de pontos com as ocorrências registradas na COPPA - 2012 Fonte: SIGAPA, 2012 - Elaboração: SANT'ANA, 2013

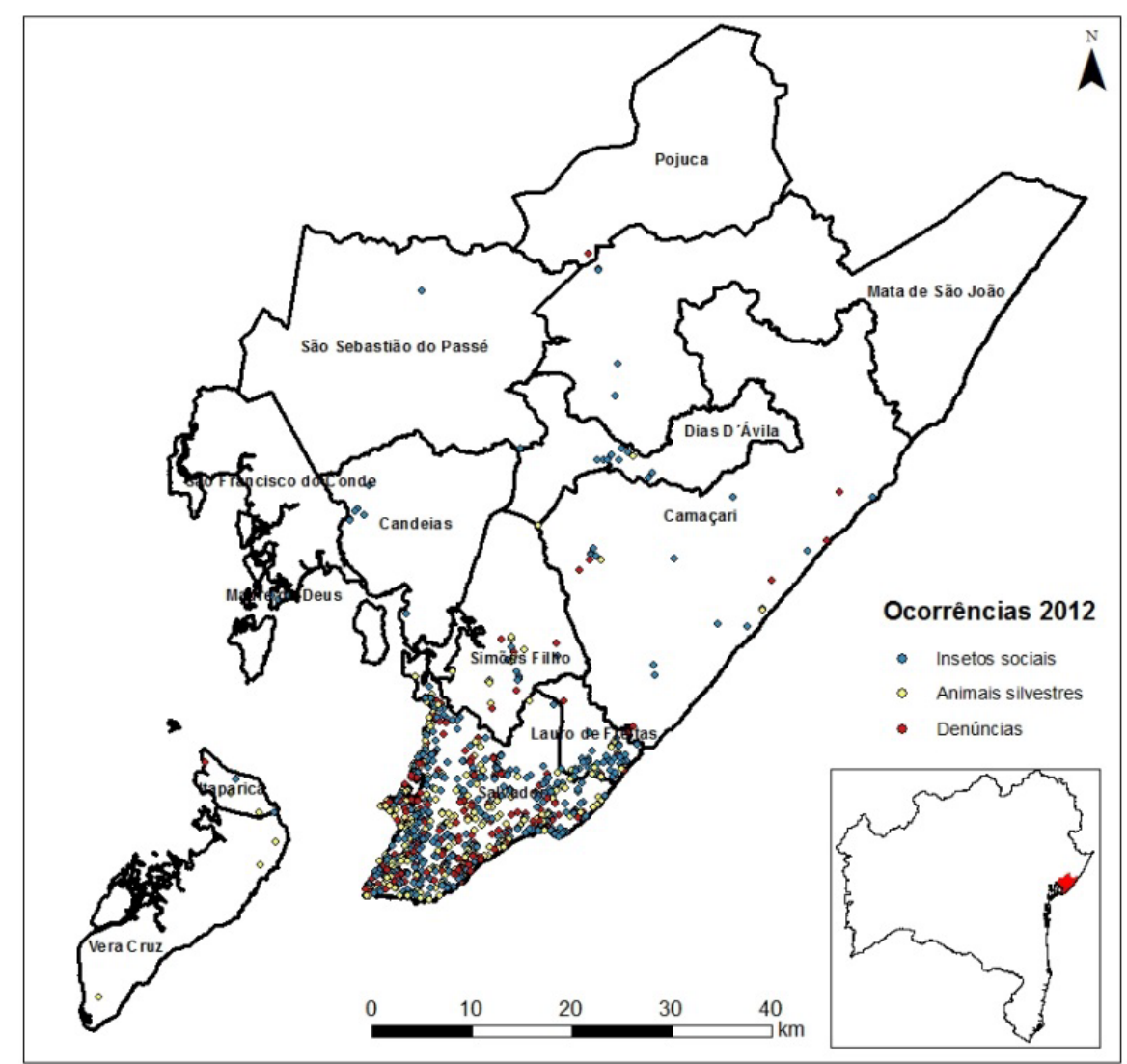


A análise de pontos permite observar por onde estão distribuídas as ocorrências. Além de Salvador que concentra 84\% das ocorrências, municípios da RMS como Lauro de Freitas (7\%), Simões Filho (2,5\%) e Camaçari (2,4\%) são os que possuem maiores registros.

A partir desse mapa de pontos, foi possível elaborar o mapa de densidade de pontos utilizando o método da densidade de Kernel. Em inglês Kernel significa “núcleo”. É utilizado para fazer estimativas de densidade e gera uma visualização mais rápida para identificar onde há uma maior concentração destes pontos. Na figura 2, é mostrada a aplicação do método Kernel para o conjunto de todas as ocorrências da COPPA em 2002.

Figura 2 - Mapa de Kernel com as ocorrências registradas na COPPA - 2012 Fonte: SIGAPA, 2012 - Elaboração: SANT'ANA, 2013

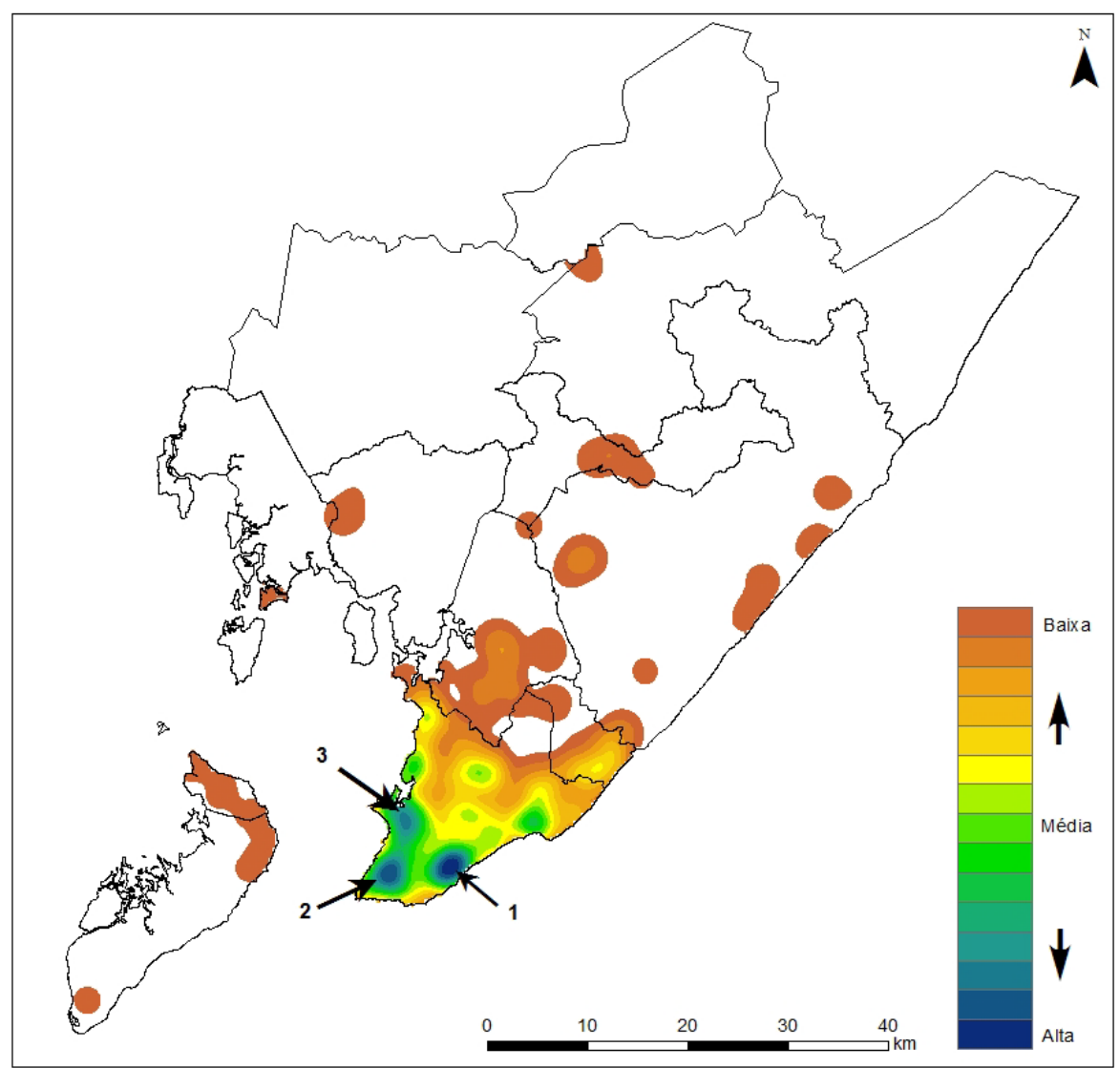

As densidades dos pontos registrados variam de baixa à alta densidade. A concentração das ocorrências está localizada mais ao sul da cidade de Salvador. Foi preferível uma divisão das três áreas, respectivamente, com a densidade mais alta. As localidades onde ocorrem mais solicitações são: (1) Stiep, Jardim Armação, Imbuí; (2) Engenho Velho de Brotas, Federação, Boa Vista de Brotas e Tororó; (3) Curuzu, Fazenda Grande do Retiro, São Caetano e Liberdade. Também em outras áreas mais isoladas como Itapuã, Cajazeiras e Plataforma onde há uma média densidade das concentrações. 
A seguir será mostrada a aplicação da densidade Kernel para cada tipo de dados de ocorrência registrado na COPPA: Insetos sociais (Figura 3), Animais silvestres (Figura 4) e Denúncias de Crimes Ambientais (Figura 5).

Das ocorrências relacionadas a ataques de insetos sociais registradas na COPPA, enxame de abelhas, marimbondos e até arapuá, são as mais comuns. São tipos de insetos que a depender da proporção e sentindo-se ameaçados, se tornam nociva a população. Em 2012, mais de 52\% dos chamados foram para capturar esses tipos de insetos.

\section{Figura 3 - Mapa de Kernel - Ocorrências dos insetos sociais - 2012} Fonte: SIGAPA, 2012 - Elaboração: SANT'ANA, 2013

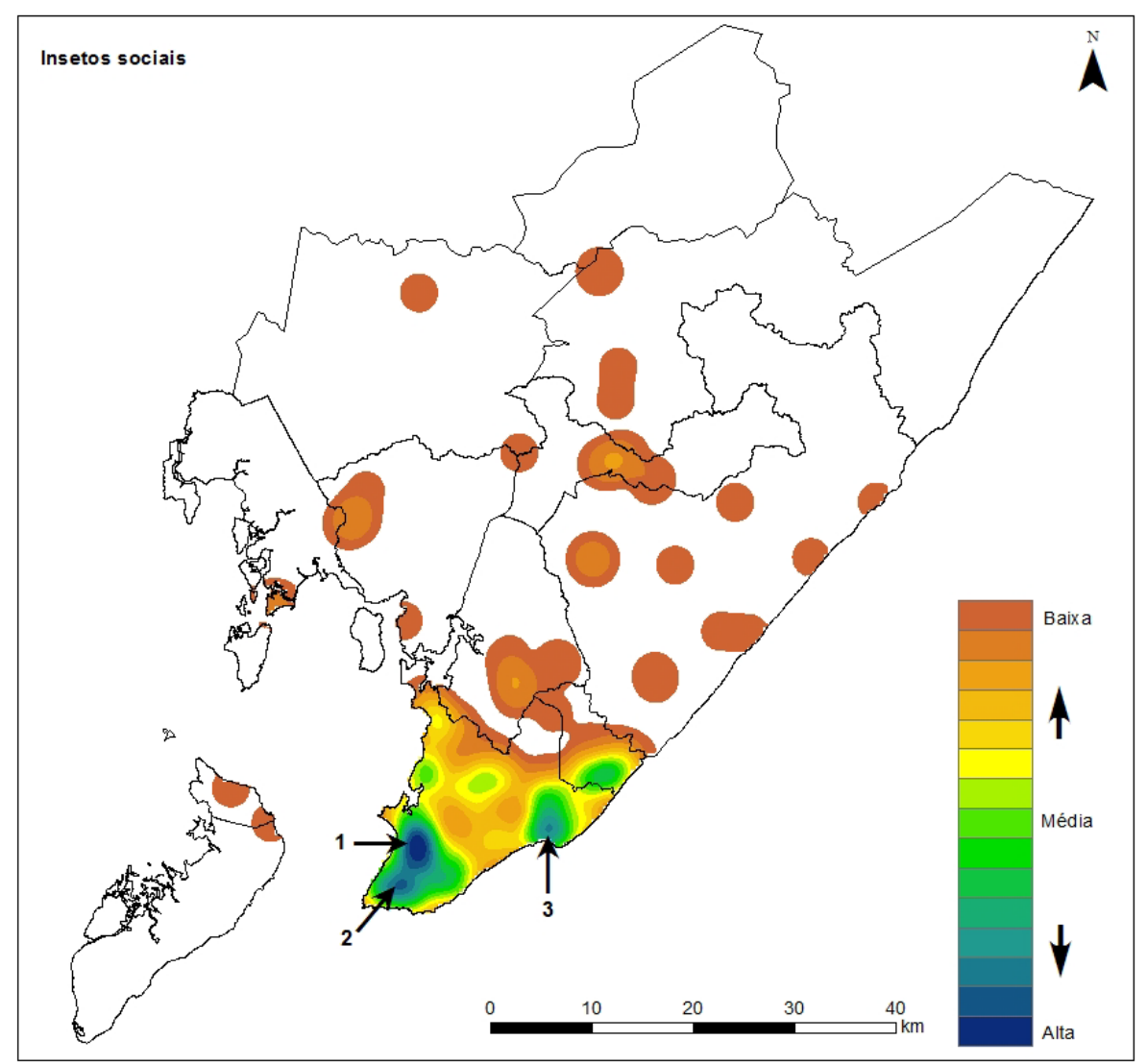

As altas densidades de insetos sociais estão mais concentradas nas localidades do (1) Iapi, Santa Mônica, Pero Vaz, Pau Miúdo, Cidade Nova, Caixa D’água; (2) Engenho Velho de Brotas e Acupe; (3) Itapuã. Também é notável a média incidência de ocorrências de insetos sociais no município de Lauro de Freitas.

A maioria das solicitações atendidas pela COPPA é para captura de abelhas. Nessas localidades citadas anteriormente, há uma grande aglomeração urbana. As abelhas, devido ao crescimento desordenado dos centros urbanos, são desalojadas e passam a ocupar áreas urbanizadas, causando alguns transtornos à população. As mais agressivas são abelhas africanizadas, que basta 
um barulho ou cheiro mais forte para elas atacarem. No verão são mais comuns. As altas temperaturas fazem com que fiquem mais agressivas. São importantes polinizadoras para preservação ambiental, pois são responsáveis pela proliferação de plantas nativas e cultivadas e aumentam a renda de alguns produtores.

Outra função que a COPPA assumiu nos últimos anos, foi o de realizar o manejo de animais silvestres encontrados em ambiente urbano.

São considerados animais silvestres (ou selvagens) todos os animais que vivem ou nascem em um ecossistema natural - como florestas, rios e oceanos. É cada vez maior o número de animais silvestres que aparecem nas cidades por causa do desmatamento e atraídos pela grande quantidade de alimentos. Em muitos bairros de Salvador tem sido comum encontrar cobras (entre jiboias e sucuris), pássaros e até raposas. Esses animais quando capturados são encaminhados para o Centro de Triagem de Animais Silvestres do IBAMA (CETAS), no bairro do Cabula, em Salvador.

Além de cobras, também micos, corujas e gaviões são os animais mais encontrados por moradores dentro de casa ou nas ruas de Salvador. Muitos animais chegam ao CETAS machucados. Os mais debilitados são aqueles apreendidos nas operações de combate ao tráfico de animais. Os animais de comportamento mais manso são levados para zoológicos ou instituições de pesquisa porque, segundo os biólogos, eles não têm condições de se alimentar sozinhos na natureza e seriam presas fáceis para outras espécies.

Na figura 5, é possível analisar os locais onde a maioria destes animais foi localizada em Salvador e RMS.

Figura 4 - Mapa de Kernel - Ocorrências de Animais Silvestres - 2012 Fonte: SIGAPA, 2012 - Elaboração: SANT'ANA, 2013

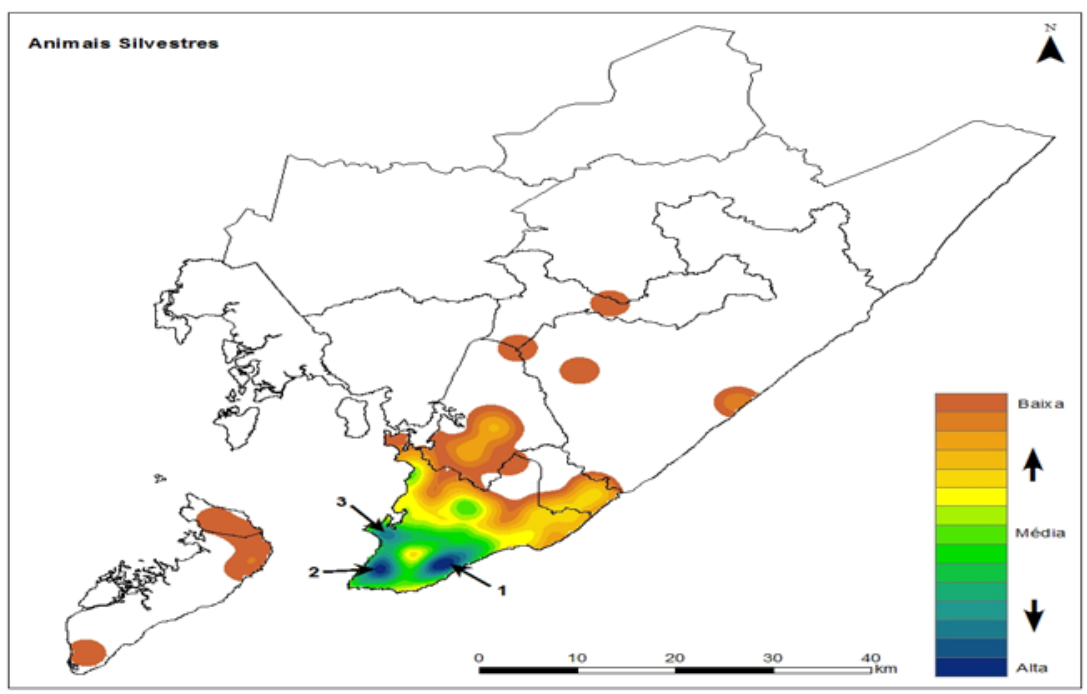


As áreas mais densas são encontradas em tons azulados, onde há maior número de ocorrências. Em (1) encontram-se as localidades do Imbuí, Stiep, Boca do Rio e Caminho das Árvores; (2) Engenho Velho de Brotas, Boa Vista de Brotas, Tororó, Nazaré e Matatu; (3) Uruguai, Vila Rui Barbosa, Calçada e São Caetano.

Algumas localidades coincidem também com áreas onde foram solicitadas atendimentos para insetos sociais. Isso contribui para hipótese do aparecimento desses animais estarem relacionadas a fatores antrópicos e ambientais.

Por fim, as denuncias são também muito comuns nas ligações recebidas pela COPPA. São moradores que delatam situações de cárceres de animais, maus tratos e venda ilegal de animais ameaçados de extinção, e também pesca com bombas.

Figura 5 - Mapa de Kernel - Denúncias de Crimes Ambientais - 2012

Fonte: SIGAPA, 2012 - Elaboração: SANT'ANA, 2013

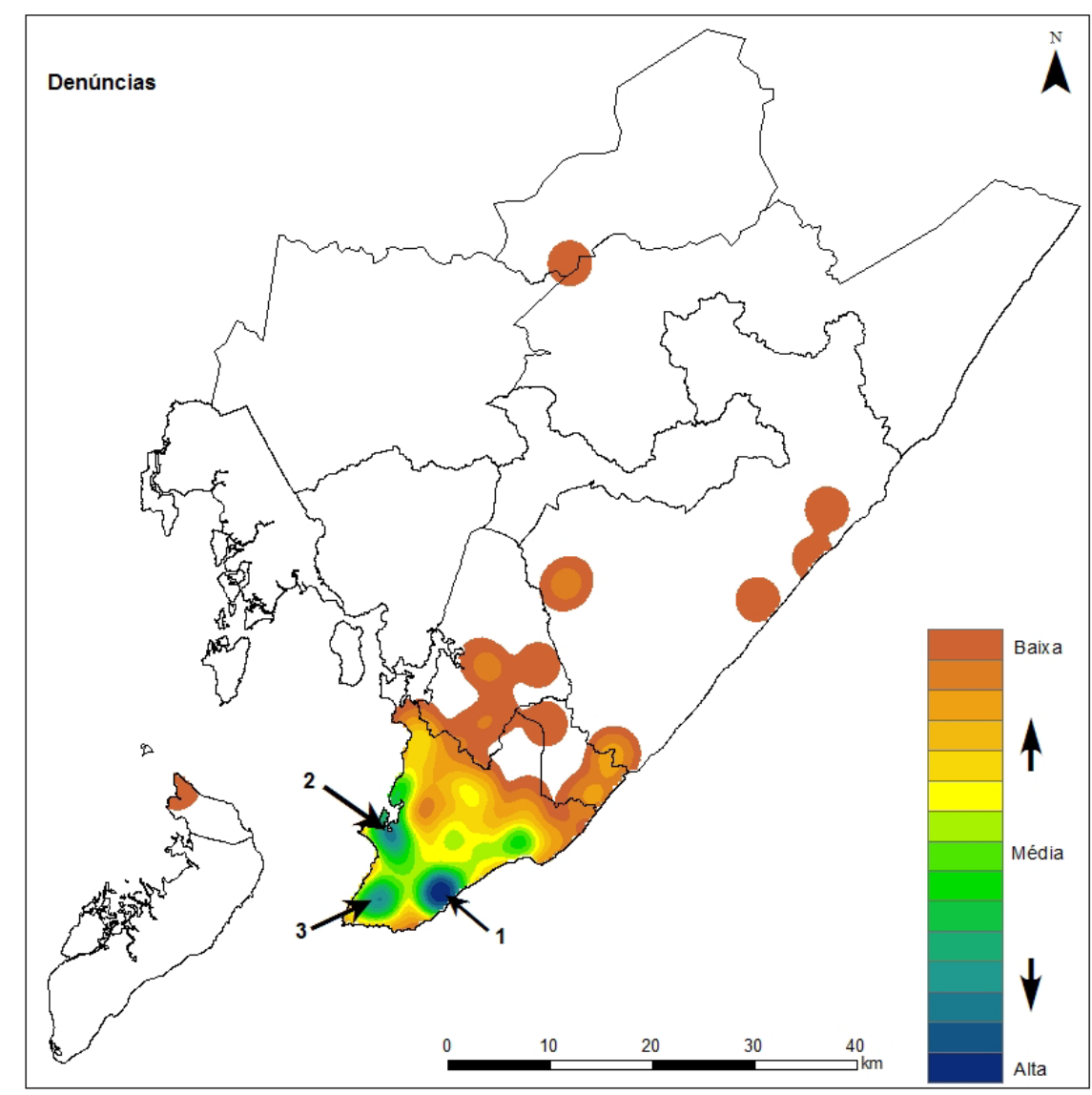

As localidades mais acionadas pela COPPA, neste caso, são: (1) Stiep, Jardim Armação, Imbuí, Boca do Rio e Caminho das Árvores; (2) Santa Luzia, Uruguai, São Caetano e Capelinha; (3) Engenho Velho de Brotas, Tororó e Boa Vista de Brotas. 
Em 2012 foram contabilizadas 183 denúncias. Esse número ainda poderia ser maior se a população denunciasse mais, pois ainda há muitos casos em que aparecem animais e são apossados pela própria população ou outros tipos de crimes ambientais e a COPPA não é informada.

\section{CONCLUSÃO}

O uso das Geotecnologias é imprescindível para a análise espacial. Elas trazem avanços significativos no desenvolvimento de pesquisas e várias outras ações de planejamento e gestão. Tanto a COPPA quanto outros segmentos que tem como objetivos desenvolver ações que beneficiem a sociedade, precisam acompanhar e incorporar esses avanços tecnológicos em suas rotinas de trabalho.

Espera-se que com a espacialização dos dados cedidos pela Companhia, sirva como apoio ao planejamento no atendimento com eficiência das demandas da população no ataque de insetos, no manejo de animas silvestres encontrados em ambiente urbano e no combate a crimes ambientais, contenção de animais, comércio ilegal e maus tratos de animais silvestres em feiras livres.

\section{AGRADECIMENTOS}

Agradecemos à UCSal e à COPPA pela disponibilidade de dados de ocorrências do ano de 2012 que foram coletados no Projeto SIGAPA, financiado pela FAPESB.

\section{REFERENCIAS}

1. BARCEllos, C.; Silva, S. A.; ANDRADE, A. L. Análise de dados em forma de pontos. In: MINISTÉRIO DA SAÚDE. Introdução à Estatistica Espacial para a Saúde Pública. Brasília: Fundação Oswaldo Cruz, p. 29-59.

2. CÂMARA, Gilberto; CARVALHO, Marilia de Sá (2004). Análise espacial de eventos. Disponivel em <http://www.dpi.inpe.br/gilberto/livro/analise/cap2-eventos.pdf $>$. Acesso em: 5 ago. 2013.

3. IPVES - Instituto de Pesquisa em Vida Selvagem. Animais silvestres são achados com frequência nas ruas e casas de Salvador. Disponível em: <http://ipevs.org.br/blog/?p=6822> . Acesso em: 12 ago. 2013.

4. SAURET, Gerard Viader. Estatísticas pela vida: a coleta e analises de informações criminais como instrumentos de enfrentamento da violência letal. Recife: Bagaço Desing, 2012. 